\title{
Scalar-Torsion Mode in a Cosmological Model of the Poincaré Gauge Theory of Gravity
}

\author{
Huan-Hsin Tseng:* Chung-Chi Lee $t^{t}$ and Chao-Qiang Gengt \\ Department of Physics, National Tsing Hua University, Hsinchu, Taiwan 300 and \\ National Center for Theoretical Sciences, Hsinchu, Taiwan 300
}

(Dated: November 5, 2018)

\begin{abstract}
We investigate the scalar-torsion mode in a cosmological model of the Poincaré gauge theory of gravity. We treat the geometric effect of torsion as an effective quantity, which behaves like dark energy, and study the effective equation of state (EoS) of the model. We concentrate on two cases with the constant curvature solution and positive kinetic energy, respectively. In the former, we find that the torsion EoS has different values in the various stages of the universe. In particular, it behaves like the radiation (matter) EoS of $w_{r}=1 / 3\left(w_{m}=0\right)$ in the radiation (matter) dominant epoch, while in the late time the torsion density is supportive for the accelerating universe. In the latter, our numerical analysis shows that in general the EoS has an asymptotic behavior in the high redshift regime, while it could cross the phantom divide line in the low redshift regime.
\end{abstract}

\footnotetext{
* d943335@oz.nthu.edu.tw

$\dagger$ g9522545@oz.nthu.edu.tw

¥ geng@phys.nthu.edu.tw
} 


\section{INTRODUCTION}

Recent cosmological observations [1 15$]$ have demonstrated that our universe is undergoing the phase of an accelerating expansion. Although general relativity (GR) developed in the last century has been successful in many ways of explaining various experimental results in gravity, the nature of the accelerating universe now rises as a small cloud shrouding it. We thereby look for a more general theory that comprises GR yet able to solve the accelerating problem referred to as dark energy [6]. By virtue of the local gauge principle, one leads to incorporate Poincaré group as gauge group of a principal bundle, such that the local Lorentz symmetry of the spacetime is preserved [7]. An attempt is to release torsion from a connection, rather than the Levi-Civita connection in the standard GR, which also acts as a dynamical field like metric tensor. Such spacetime is usually called Riemann-Cartan manifold. The gauge theory based on this manifold, is known as Poincaré gauge theory (PGT) [8 11].

It has been investigated in [10, 12] that there are six modes by the decomposition of the connection according to torsion tensor in the linearlized theory, classified as $0^{ \pm}, 1^{ \pm}$and $2^{ \pm}$ in terms of spins and parities. Among them, the $0^{+}$mode [13], also called the scalar-torsion mode, does not directly interact with any known fundamental source [14]. Along with the property induced by the nonlinear equation set, this $0^{+}$mode is our main concern. In [14], Shie, Nester and Yo (SNY) have examined models with the spin $0^{+}$mode in PGT to achieve the late time accelerating expansion of the universe. In other words, the geometric effect of torsion is treated as an effective dark energy. In particular, they have presented two cases with the solutions of a constant curvature and positive kinetic energy, respectively. The first case is an extremely simple solution existing inside the system of differential equations formed by the spin $0^{+}$mode, which provides a modeling for the late time de Sitter universe for dark energy. Note that this simple solution violates the positivity argument [14]. The second one is referred to as the normal case, which conforms with the regular positivity condition, but it gives rise to no obvious analytic solution. Torsion cosmology related to the scalar-torsion mode has been also explored in [15 23]. In this work, we concentrate on these two cases and present numerical solutions of the late-time acceleration behavior corresponding to the equation of state (EoS), defined by $w=p / \rho$, where $\rho$ and $p$ are the energy density and pressure of the relevant component of the universe, respectively. 
This paper is organized as follows: In Sec. II, we review the scalar-torsion of the spin $0^{+}$mode in PGT and give equations of motion for cosmology. In Sec. III, we show our numerical results on the cosmological evolutions for the scalar-torsion mode. We present our conclusions in Sec. IV.

\section{SCALAR-TORSION MODE IN POINCARÉ GAUGE THEORY}

\section{A. Lagrangian for the scalar-torsion mode}

PGT of gravity starts with a Lagrangian 4-form on $U_{4}$-spacetime:

$$
\mathcal{L}(g, \vartheta, \Gamma)=\mathcal{L}_{G}+\mathcal{L}_{M}
$$

where $\left\{\vartheta^{i}\right\}$ is a set of the orthonormal dual basis, $\Gamma_{j}{ }^{i}$ is the connection 1-form with respect to $\left\{\vartheta^{i}\right\}, \mathcal{L}_{M}$ is the matter Lagrangian, and $\mathcal{L}_{G}$ is the gravitational Lagrangian that can be made up by certain combinations. In [14], SNY studied the spin $0^{+}$mode, given by [14, 24]

$$
\mathcal{L}_{G}=\frac{a_{0}}{2} R \eta+\frac{b}{24} R^{2} \eta+\frac{a_{1}}{8}\left({ }^{(1)} T^{i} \wedge \star^{(1)} T_{i}\right)
$$

where $\star$ is the Hodge dual map, $\eta$ is the volume 4 -form of the space-time and ${ }^{(J)} T^{i}$ with $J=1,2,3$ are the irreducible pieces of the torsion 2 -form $T^{i}=d \vartheta^{i}+\Gamma_{j}{ }^{i} \wedge \vartheta^{j}$, defined by [11]

$$
{ }^{(1)} T^{i}=T^{i}-{ }^{(2)} T^{i}-{ }^{(3)} T^{i}, \quad{ }^{(2)} T^{i}=\frac{1}{3} \vartheta^{i} \wedge\left(i_{e_{j}} T^{j}\right), \quad{ }^{(3)} T^{i}=\frac{1}{3} \star\left(\vartheta^{i} \wedge \star\left(T^{j} \wedge \vartheta_{j}\right)\right) .
$$

The coefficients of $\mathcal{L}_{G}$ in (2) are constrained by the positivity argument [14] such that

$$
a_{1}>0, \quad b>0
$$

The independent variation of (11) with respect to $\left(g_{i j}, \vartheta^{i}, \Gamma_{j}{ }^{i}\right)$ yields [25]

$$
\begin{aligned}
\delta \mathcal{L}_{G} & =\frac{1}{2} K^{i j} \delta g_{i j}+E_{i} \wedge \delta \vartheta^{i}+E_{i}{ }^{j} \wedge \delta \Gamma_{j}{ }^{i}+\text { an exact form, } \\
\delta \mathcal{L}_{M} & =\frac{1}{2} T^{i j} \delta g_{i j}+t_{i} \wedge \delta \vartheta^{i}+s_{i}{ }^{j} \wedge \delta \Gamma_{j}{ }^{i}+\text { an exact form, }
\end{aligned}
$$

where the gauge field momenta are given by

$$
K^{i j}=2 \frac{\delta \mathcal{L}_{G}}{\delta g_{i j}}, \quad E_{i}=\frac{\delta \mathcal{L}_{G}}{\delta \vartheta^{i}}, \quad E_{i}^{j}=\frac{\delta \mathcal{L}_{G}}{\delta \Gamma_{j}{ }^{i}},
$$


and the source terms are defined by

$$
T^{i j}=2 \frac{\delta L_{M}}{\delta g_{i j}}, \quad t_{i}=\frac{\delta L_{M}}{\delta \vartheta^{i}}, \quad s_{i}{ }^{j}=\frac{\delta L_{M}}{\delta \Gamma_{j}{ }^{i}},
$$

corresponding to the symmetric energy-momentum tensor-valued 4-form, asymmetric vectorvalued 3-form usually called canonical energy-momentum tensor, and tensor-valued 3-form known as canonical spin angular momentum tensor, respectively. One can also write the decompositions into the basis of $\Omega(M)$ [24, 25]:

$$
t_{i}=\mathscr{T}_{i k} \eta^{k}, \quad s_{i j}=S_{i j k} \eta^{k}
$$

where $\eta^{k}:=\star \vartheta^{k}$. The equations of motion are given symbolically as

$$
E_{i}=-t_{i}, \quad E_{i j}=-s_{i j}
$$

\section{B. Equations of motion for cosmology}

We shall describe our universe with the FLRW cosmology, which is homogeneous and isotropic with the metric

$$
d s^{2}=-d t^{2}+a^{2}(t)\left(\frac{d r^{2}}{1-k r^{2}}+r^{2} d \Omega^{2}\right),
$$

where $k$ is the constant curvature. For simplicity, we shall only consider the flat universe with $k=0$.

For (2) of the SNY model in the FRLW cosmology with no spin source $\left(S_{i j k} \equiv 0\right)$, the main field equation (9]) leads to [14]

$$
\begin{array}{r}
\dot{H}=\frac{\mu}{6 a_{1}} R+\frac{1}{6 a_{1}} \mathscr{T}-2 H^{2}, \\
\dot{\Phi}(t)=\frac{a_{0}}{2 a_{1}} R+\frac{\mathscr{T}}{2 a_{1}}-3 H \Phi+\frac{1}{3} \Phi^{2}, \\
\dot{R}=-\frac{2}{3}\left(R+\frac{6 \mu}{b}\right) \Phi,
\end{array}
$$

where $\mu=a_{1}+a_{0}, H=\dot{a}(t) / a(t)$, and $\Phi(t)=T_{t}$, which is the time component of the torsion trace, defined by $T_{i}=T_{i j}{ }^{j}$. Here, $R$ in (11)-(13) denotes the affine curvature with respect to the curvature 2 -form $\Omega_{i}{ }^{j}$, given by

$$
\Omega_{i}^{j}=d \Gamma_{i}^{j}+\Gamma_{k}^{j} \wedge \Gamma_{i}^{k}=\frac{1}{2} R^{j}{ }_{i k l} \vartheta^{k} \wedge \vartheta^{l} .
$$


Hence, one obtains the relation

$$
R=\bar{R}+2 \frac{\partial T^{j}}{\partial x^{j}}-\frac{2}{3} T_{k} T^{k}
$$

where $\bar{R}=6\left(\dot{H}+2 H^{2}\right)$ represents the curvature of the Levi-Civita connection induced by (10). The energy-momentum tensor $\mathscr{T}_{i j}$ is defined as (8) and $\mathscr{T}$ stands for the trace $\mathscr{T}_{i}{ }^{i}$. Explicitly, one has

$$
\begin{aligned}
\mathscr{T}_{t t} & =\rho_{M}=\frac{b}{18}\left(R+\frac{6 \mu}{b}\right)(3 H-\Phi)^{2}-\frac{b}{24} R^{2}-3 a_{1} H^{2}, \\
\mathscr{T} & =3 p_{M}-\rho_{M} .
\end{aligned}
$$

with the subscript $M$ representing the ordinary matter including both dust and radiation.

To see the geometric effect of torsion, we can write down the Friedmann equations as

$$
\begin{aligned}
H^{2} & =\frac{\rho_{c}}{3 a_{0}}, & \rho_{c} & =\rho_{M}+\rho_{T}, \\
\dot{H} & =-\frac{\rho_{c}+p_{t o t}}{2 a_{0}}, & p_{t o t} & =p_{M}+p_{T},
\end{aligned}
$$

with $a_{0}=(8 \pi G)^{-1}$ in GR, where $\rho_{c}$ and $p_{\text {tot }}$ denote the critical energy density and total pressure of the universe, while $\rho_{T}$ and $p_{T}$ correspond to the energy density and pressure of some effective field, respectively. By comparing the equation of motion of the scalar-torsion mode in PGT (16) to the Friedmann equations (17), one obtains

$$
\begin{aligned}
\rho_{T} & =3 \mu H^{2}-\frac{b}{18}\left(R+\frac{6 \mu}{b}\right)(3 H-\Phi)^{2}+\frac{b}{24} R^{2}, \\
p_{T} & =\frac{1}{3}\left(\mu(R-\bar{R})+\rho_{T}\right),
\end{aligned}
$$

which will be regarded as the effective torsion dark energy density and pressure, respectively. From (17), we get the continuity equation,

$$
\dot{\rho}_{c}+3 H\left(\rho_{c}+p_{t o t}\right)=0,
$$

which can also be derived by applying the identity

$$
\bar{\nabla}_{j} \bar{G}^{i j}=\bar{\nabla}_{j}\left(\bar{R}^{i j}-\frac{1}{2} \bar{R} g^{i j}\right)=\bar{\nabla}_{j}\left(\mathscr{T}^{i j}+\mathscr{T}_{T}^{i j}\right)=0,
$$

where $\bar{\nabla}$ is the covariant derivative with respect to the Levi-Civita connection and $\mathscr{T}_{T}{ }^{i}{ }_{j}=$ $\operatorname{diag}\left(-\rho_{T}, p_{T}, p_{T}, p_{T}\right)$ is the effective energy-momentum tensor of torsion dark energy. 
In addition, from (11) - (13), one can check that the continuity equation for the torsion field is also valid, i.e.

$$
\dot{\rho}_{T}+3 H\left(\rho_{T}+p_{T}\right)=0 .
$$

Consequently, we obtain the continuity equation for the ordinary matter to be

$$
\dot{\rho}_{M}+3 H\left(\rho_{M}+p_{M}\right)=0 .
$$

By assuming no coupling between radiation and dust, the matter densities of radiation $\left(w_{r}=\right.$ $1 / 3)$ and dust $\left(w_{m}=0\right)$ in scalar-torsion cosmology share the same evolution behaviors as GR, i.e. $\rho_{r} \propto a^{-4}$ and $\rho_{m} \propto a^{-3}$, respectively. In order to investigate the cosmological evolution, it is natural to define the total EoS by [6]

$$
w_{t o t}=-1-\frac{2 \dot{H}}{3 H^{2}}=\frac{p_{t o t}}{\rho_{c}},
$$

which leads to

$$
w_{t o t}=\Omega_{M} w_{M}+\Omega_{T} w_{T},
$$

where $\Omega_{\alpha}=\rho_{\alpha} / \rho_{c}$ and $w_{\alpha}=p_{\alpha} / \rho_{\alpha}$ with $\alpha=M, T$, representing the energy density ratios and EoSs of matter and torsion, respectively. Note that the EoS in (23), which is commonly used in the literature, e.g. [6], can be examined by cosmological observations in [1 [5]. In particular, it can be used to distinguish the modified gravity theories from $\Lambda$ CDM [6]. Consequently, the evolution of the torsion dark energy can be described in terms of solely $w_{T}$ by

$$
\rho_{T}(z)=\rho_{T}^{(0)} \exp \left\{3 \int_{0}^{z} d z^{\prime} \frac{1+w_{T}\left(z^{\prime}\right)}{1+z^{\prime}}\right\} .
$$

In the following section, we shall focus on this important quantity.

\section{NUMERICAL RESULTS OF TORSION COSMOLOGY}

The evolution of torsion cosmology is determined by (11) - (13). In general, one needs to solve the dynamics of $R, \Phi$ and $H$ by the system of ordinary differential equations. However, one easily sees that in (13) there exists a special case, in which the constant scalar affine curvature $R=-6 \mu / b$ is a possible solution [14]. Recall that in order to conform with the positive kinetic energy argument, the condition (3) is needed. However, since the special 
case yields the negative curvature $R=-6 \mu / b<0$ with a negative matter density $\rho<0$, the condition of $a_{1}<-a_{0}<0$ is required [14].

In this section, we concentrate on the EoS of the scalar-torsion mode in both special and normal cases. We also present the cosmological evolution of the density ratio, defined by $\Omega=\rho / \rho_{c}$, from a high redshift to the current stage.

\section{A. Special Case: $R=$ const.}

In this special case, we take the assumptions of $a_{1}, \mu<0$ and $a_{0}>0$ in [14]. The evolution equations (11) - (13) reduce to

$$
\begin{aligned}
\rho_{M} & =-3 a_{1} H^{2}-\frac{3}{2} \frac{\mu^{2}}{b}, \\
\rho_{T} & =\frac{3}{2} \frac{\mu^{2}}{b}+3 \mu H^{2}, \\
\dot{H} & =-\left(1+w_{M}\right)\left(\frac{3}{4} \frac{\mu^{2}}{a_{1} b}+\frac{3}{2} H^{2}\right) .
\end{aligned}
$$

For the numerical calculation, we rescale the parameters as follows:

$$
\begin{aligned}
m^{2} & =\rho_{m}^{(0)} / 3 a_{0}, \quad \tilde{a}_{0}=a_{0} / m^{2} b, \quad \tilde{a}_{1}=-a_{1} / m^{2} b, \\
\tilde{t} & =m \cdot t, \quad \tilde{\mu}=\tilde{a}_{1}-\tilde{a}_{0}, \quad \tilde{H}^{2}=H^{2} / m^{2}, \quad \tilde{R}=R / m^{2},
\end{aligned}
$$

where $\rho_{m}^{(0)}$ is the matter density at $z=0$ and the scalar affine curvature is a constant $\tilde{R}=6 \tilde{\mu}>0$. From (25), (26) and (27), we obtain the following dimensionless equations,

$$
\begin{aligned}
& \tilde{H}^{2}=\frac{\tilde{a}_{0}}{\tilde{a}_{1}}\left(a^{-3}+\chi a^{-4}\right)+\frac{\tilde{\mu}^{2}}{2 \tilde{a}_{1}}, \\
& \frac{\rho_{T}}{\rho_{m}^{(0)}}=\frac{\tilde{\mu}^{2}}{2 \tilde{a}_{0}}-\frac{\tilde{\mu}}{\tilde{a}_{0}} \tilde{H}^{2}, \\
& \tilde{H} \tilde{H}^{\prime}=\left(1+w_{M}\right)\left(\frac{3}{4} \frac{\tilde{\mu}^{2}}{\tilde{a}_{1}}-\frac{3}{2} \tilde{H}^{2}\right),
\end{aligned}
$$

where the prime " $\prime$ " stands for $d / d \ln a$ and $\chi=\rho_{r}^{(0)} / \rho_{m}^{(0)}$. Using (20), (23) and (130), we find that

$$
w_{T}=-1-\frac{\dot{\rho}_{T}}{3 H \rho_{T}}=-1-\frac{4}{3} \frac{\dot{\tilde{H}}}{2 \tilde{H}^{2}-\tilde{\mu}} .
$$



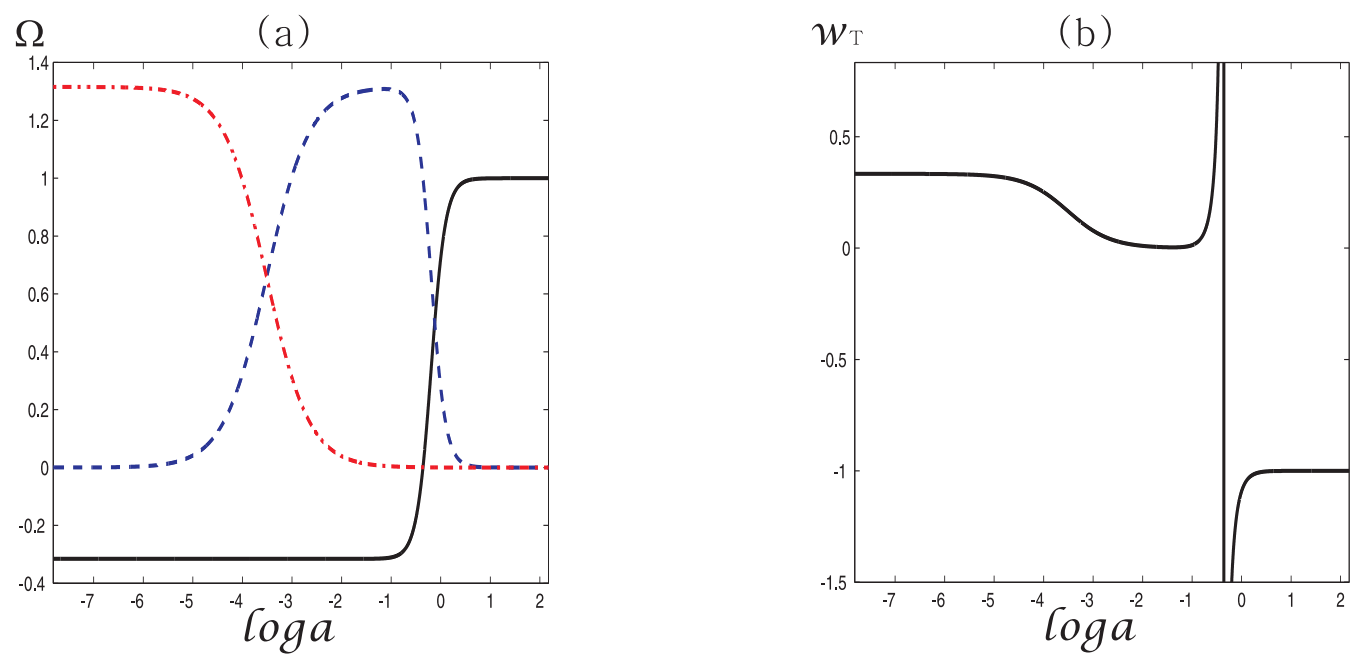

Figure 1. Evolutions of (a) the energy density ratio $\Omega$ and (b) the torsion $\operatorname{EoS} w_{T}$ with $\Omega_{m}^{(0)}=$ 27.5\%, where the solid (black), dashed (blue), and dotted-dashed (red) lines stand for torsion, matter and radiation, respectively.

From (29)-(32), it is easy to see that the evolution of $\rho_{T}$ is automatically determined without solving any differential equation for given values of $\tilde{a}_{0}$ and $\tilde{a}_{1}$. The numerical results of this special case are shown in Fig 1, where we have chosen $\tilde{a}_{0}=76, \tilde{a}_{1}=100$ and $\chi=3.07 \times 10^{-4}$ in corresponding to $\Omega_{m}^{(0)}=\tilde{H}_{z=0}^{-2} \simeq 27.5 \%$. In Fig. 1ha, we plot the energy density ratios of torsion, matter and radiation, $\Omega_{T}, \Omega_{m}$ and $\Omega_{r}$, respectively. Notice that $\rho_{T}$ depends on the parameters $\tilde{a}_{0}$ and $\tilde{a}_{1}$, and there exists a late-time de-Sitter solution when $\tilde{H}^{2}=\tilde{\mu}^{2} / 2 \tilde{a}_{1}$. In the high redshift regime, in which $\tilde{H}^{2} \gg \tilde{\mu}, \tilde{\mu}^{2} / \tilde{a}_{1}$, we observe that the torsion density ratio $\Omega_{T}$ is a constant which can also be estimated from (25) and (26), namely

$$
\frac{\rho_{M}}{\rho_{T}}=\frac{3 \tilde{a}_{1} \tilde{H}^{2}-3 \tilde{\mu}^{2} / 2}{3 \tilde{\mu}^{2} / 2-3 \tilde{\mu} \tilde{H}^{2}} \simeq-\frac{\tilde{a}_{1}}{\tilde{\mu}},
$$

which manifests itself a negative constant. In Fig. 1 $\mathrm{b}$, we show that the torsion EoS $w_{T}$ acts as matter $w_{m}=0$ and radiation $w_{r}=1 / 3$ in the matter-dominant $\left(\rho_{m} \gg \rho_{r}\right)$ and radiationdominant $\left(\rho_{r} \gg \rho_{m}\right)$ stages, respectively, which are interesting asymptotic behaviors. We also observe that in the low redshift regime of $\log a \simeq 0, w_{T}$ is smaller than unity, indicating the existence of a late-time acceleration epoch. 


\section{B. Normal Case}

The normal case here denotes the positive definiteness of both kinetic energy and matter density, i.e, the parameters $a_{0}, a_{1}$ and $b$ are subject to the condition (3). It is also convenient to rescale the parameters such that

$$
\begin{aligned}
\tilde{a}_{0}=a_{0} / m^{2} b, & \tilde{a}_{1}=a_{1} / m^{2} b, \quad \tilde{t}=t \cdot m, \quad \tilde{\mu}=\tilde{a}_{0}+\tilde{a}_{1}, \\
\tilde{H}^{2}=H^{2} / m^{2}, & \tilde{\Phi}=\Phi / m, \quad \tilde{R}=R / m^{2},
\end{aligned}
$$

where $m^{2}=\rho_{m}^{(0)} / 3 a_{0}$. Using the above rescaling parameters, (11) - (13) and (16) are then rewritten as

$$
\begin{aligned}
& \tilde{H} \tilde{H}^{\prime}=\frac{\tilde{\mu}}{6 \tilde{a}_{1}} \tilde{R}-\frac{\tilde{a}_{0}}{2 \tilde{a}_{1}} a^{-3}-2 \tilde{H}^{2} \\
& \tilde{H} \tilde{\Phi}^{\prime}=\frac{\tilde{a}_{0}}{2 \tilde{a}_{1}}\left(\tilde{R}-3 a^{-3}\right)-3 \tilde{H} \tilde{\Phi}+\frac{1}{3} \tilde{\Phi}^{2} \\
& \tilde{H} \tilde{R}^{\prime}=-\frac{2}{3}(\tilde{R}+6 \tilde{\mu}) \tilde{\Phi} \\
& \frac{1}{18}(\tilde{R}+6 \tilde{\mu})(3 \tilde{H}-\tilde{\Phi})-\frac{\tilde{R}^{2}}{24}-3 \tilde{a}_{1} \tilde{H}^{2}=3 \tilde{a}_{0}\left(a^{-3}+\chi a^{-4}\right),
\end{aligned}
$$

where we have used $\stackrel{e m}{T}=3 P_{M}-\rho_{M}=-\rho_{m}=-3 a_{0} m^{2} a^{-3}$ due to $w_{r}=p_{r} / \rho_{r}=1 / 3$ and $w_{m}=p_{m} / \rho_{m}=0$. From (23) and (35)-(38), we have

$$
w_{T}=\frac{1}{3} \frac{\tilde{\mu}\left(\tilde{R}-\bar{R} / m^{2}\right)}{3 \tilde{\mu} \tilde{H}^{2}-(\tilde{R}+6 \tilde{\mu})(3 \tilde{H}-\tilde{\Phi})^{2} / 18+\tilde{R}^{2} / 24}+\frac{1}{3} .
$$

To perform the numerical computations, we need to specify two parameters: $\tilde{a}_{0}$ and $\tilde{a}_{1}$, along with two initial conditions: $\tilde{R}$ and $\tilde{H}$. Thus, the initial condition for $\tilde{\Phi}$ is automatically determined by (38). The numerical results are shown in Fig. 2, where the initial conditions at $z=0$ are set as $\left(\tilde{a}_{0}, \tilde{a}_{1}, \tilde{R}_{0}, \tilde{H}_{0}\right)=(2,1,14,2),(2,1,13,2),(3,1,8,2)$ for solid, dot-dashed, and dashed lines, respectively. Note that $\chi=3.07 \times 10^{-4}$ originates from the WMAP-5 data, and $\tilde{H}=2$ corresponds to $\Omega_{m}^{(0)}=\tilde{H}_{0}^{-2}=0.25$.

In Fig. 2 a, we show the evolution of the density ratio, $\Omega_{T}=\rho_{T} / \rho_{c}$, as a function of the redshift $z$. The figure demonstrates that the torsion density $\rho_{T}$ dominates the universe in the high redshift regime $(z \gg 1)$ with the general parameter and initial condition selection, while the matter-dominated regime is reached only within a very short time interval. In Fig. 2b, 

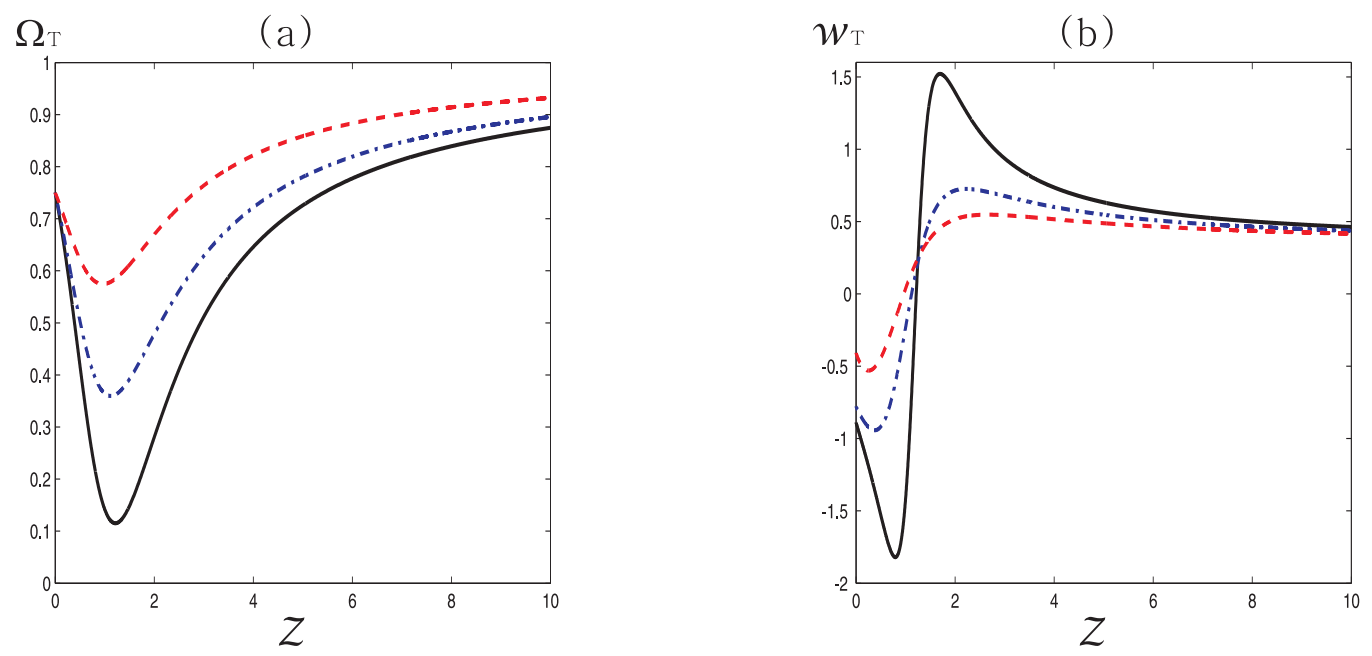

Figure 2. Evolutions of (a) the energy density ratio $\Omega_{T}$ and (b) the torsion $\operatorname{EoS} w_{T}$ in the universe as functions of the redshift $z$ with $\Omega_{m}^{(0)}=25 \%$ and $\chi=3.07 \times 10^{-4}$, where the solid, dotted-dashed and dashed lines correspond to $\left(\tilde{a}_{0}, \tilde{a}_{1}, \tilde{R}_{0}, \tilde{H}_{0}\right)=(2,1,14,2),(2,1,13,2),(3,1,8,2)$, respectively.

we show that $w_{T}$ has an asymptotic behavior at the high redshift regime, i.e. $w_{z \gg 0} \rightarrow 1 / 3$. Moreover, in the low redshift regime, it may even have a phantom crossing behavior, i.e., the torsion EoS could cross the phantom divide line of $w_{T}=-1$. As a result, the scalar-torsion mode is able to account for the late-time accelerating universe. Finally, we remark that the studies in Refs. [14, 18] only indicated an oscillating behavior without the asymptotic one above for the torsion density.

\section{CONCLUSIONS}

We have studied the torsion EoS of the two cases of the scalar-torsion mode in PGT of gravity, which are suitable for explaining the late-time accelerating universe but each of them possesses a quite different cosmological behavior in the high redshift regime. For the first case, which violates the positive kinetic energy and has a constant affine curvature $R$, the torsion EoS has asymptotic behaviors: $w_{T}=1 / 3$ in the radiation-dominated stage, $w_{T}=0$ in the matter-dominated stage, and finally a late-time de-Sitter solution corresponding to $w_{T}=-1$. The torsion density ratio of $\Omega_{T}$ in the high redshift regime is a "negative" constant. For the second one, which has the positive kinetic energy, under the general selection of parameters and initial conditions, the torsion EoS still shows an asymptotic behavior, $w=1 / 3$, in the high redshift regime, while it could cross the phantom divide line 
in the low redshift regime. The most confusing phenomenon in this spin $0^{+}$scalar-torsion cosmology is that the universe is dominated by torsion in the high redshift regime even though there exists a narrow window for the matter-dominated epoch.

\section{ACKNOWLEDGMENTS}

We are grateful to Professor H. J. Yo and Professor J. M. Nester for the inspiring and helpful discussions. This work was partially supported by National Center of Theoretical Science, National Tsing Hua University and National Science Council (NSC-98-2112-M-007008-MY3) of R.O.C.

[1] A. G. Riess et al. [Supernova Search Team Collaboration], Astron. J. 116, 1009 (1998).

[2] S. Perlmutter et al. [Supernova Cosmology Project Collaboration], Astrophys. J. 517, 565 (1999).

[3] D. N. Spergel et al. [WMAP Collaboration], Astrophys. J. Suppl. 148, 175 (2003).

[4] M. Tegmark et al. [SDSS Collaboration], Phys. Rev. D69, 103501 (2004).

[5] S. W. Allen, R. W. Schmidt, H. Ebeling, A. C. Fabian, L. van Speybroeck, Mon. Not. Roy. Astron. Soc. 353, 457 (2004).

[6] E. J. Copeland, M. Sami and S. Tsujikawa, Int. J. Mod. Phys. D 15, 1753 (2006); M. Li, X. -D. Li, S. Wang and Y. Wang, Commun. Theor. Phys. 56, 525 (2011).

[7] F. W. Hehl, P. Von Der Heyde, G. D. Kerlick and J. M. Nester, Rev. Mod. Phys. 48, 393 (1976).

[8] Y. N. Obukhov, V. N. Ponomarev and V. V. Zhytnikov, Gen. Rel. Grav. 21, 1107 (1989).

[9] F. W. Hehl, "Four Lectures On Poincare Gauge Field Theory," in Proc. of the 6th Course of the School of Cosmology and Gravitation on Spin, Torsion, Rotation, and Supergravity, held at Erice, Italy, 1979 (P. G. Bergmann and V. De Sabbata, eds.), p. 5. Plenum, 1980.

[10] K. Hayashi and T. Shirafuji, Prog. Theor. Phys. 64, 866 (1980); 64, 883 (1980); 64, 1435 (1980); 64, 2222 (1980); 65, 525 (1981); 66, 318 (1981); 66, 2258 (1981).

[11] F. W. Hehl, J. D. McCrea, E. W. Mielke and Y. Ne'eman, Phys. Rept. 258, 1 (1995).

[12] E. Sezgin and P. van Nieuwenhuizen, Phys. Rev. D 21, 3269 (1980). 
[13] W. Kopczyński, Phys. Lett. A 39, 219 (1972)

[14] K. F. Shie, J. M. Nester and H. J. Yo, Phys. Rev. D 78, 023522 (2008).

[15] H. Chen, F. -H. Ho, J. M. Nester, C. -H. Wang and H. -J. Yo, JCAP 0910, 027 (2009).

[16] X. -z. Li, C. -b. Sun and P. Xi, Phys. Rev. D 79, 027301 (2009).

[17] X. -z. Li, C. -b. Sun and P. Xi, JCAP 0904, 015 (2009).

[18] X. -c. Ao, X. -z. Li and P. Xi, Phys. Lett. B 694, 186 (2010).

[19] P. Baekler, F. W. Hehl and J. M. Nester, Phys. Rev. D 83, 024001 (2011).

[20] F. -H. Ho and J. M. Nester, J. Phys. Conf. Ser. 330, 012005 (2011).

[21] X. -c. Ao and X. -z. Li, JCAP 1202, 003 (2012).

[22] F. -H. Ho and J. M. Nester, Annalen Phys. 524, 97 (2012).

[23] P. Xi, X. -h. Zhai and X. -z. Li, Phys. Lett. B 706, 482 (2012).

[24] F. W. Hehl, arXiv:1204.3672 [gr-qc].

[25] A. Trautman, arXiv:0606062 [gr-qc]. 